\title{
A Positive-Weight Next-to-Leading-Order Monte Carlo for Heavy Flavour Hadroproduction
}

\author{
Stefano Frixione \\ INFN, Sezione di Genova, Italy \\ E-mail: Stefano.Frixione@ge.infn.it \\ Paolo Nason \\ INFN, Sezione di Milano Bicocca, Italy \\ E-mail: Paolo.Nason@mib.infn.it \\ Giovanni Ridolfi \\ Dipartimento di Fisica, Università di Genova \\ and INFN, Sezione di Genova, Italy \\ E-mail: Giovanni.Ridolfi@ge.infn.it
}

\begin{abstract}
We present a next-to-leading order calculation of heavy flavour production in hadronic collisions that can be interfaced to shower Monte Carlo programs. The calculation is performed in the context of the POWHEG method [1]. It is suitable for the computation of charm, bottom and top hadroproduction. In the case of top production, spin correlations in the decay products are taken into account.
\end{abstract}




\section{Contents}

1. Introduction 1

2. Kinematics and cross section $\quad 2$

2.1 Born and Soft-Virtual kinematics 2

2.2 Real emission kinematics 4

2.3 Collinear remnants 6

$\begin{array}{lll}\text { 3. Cross section for the hardest emission } & 7\end{array}$

4. Generation of the hardest event 9

5. Accuracy of the Sudakov form factor 9

6. Colour assignment 9

$\begin{array}{ll}\text { 7. Results } & 10\end{array}$

$\begin{array}{lll}\text { 8. Conclusions } & 15\end{array}$

\section{Introduction}

The study of heavy flavoured hadronic final states produced in hadron collisions has received great attention in the past twenty years. This interest was driven partly by the search for the top quark, and partly by the circumstance that processes characterized by one single large energy scale (the heavy quark mass in this case) are reasonably controlled by perturbative QCD due to asymptotic freedom. Theoretical progress has therefore parallelled the experimental efforts in this direction, and has by now reached a remarkable level of refinement.

The next-to-leading order (NLO) calculation of heavy flavour hadroproduction has been matched to the HERWIG SMC generator $[2,3]$ in the context of the MC@NLO formalism $[4,5]$. This development is particularly important, since it has always proved difficult to correctly simulate heavy flavour production in standard shower Monte Carlo programs (SMC from now on), because of the impact of higher order processes like flavour excitation and gluon splitting.

In ref. [1] a method for interfacing NLO calculations with SMC generators was suggested, that overcomes some drawbacks of the MC@NLO technique. In particular, the implementation of a given production process with the method of ref. [1] is independent of the SMC it will be matched to, whereas this is not the case of MC@NLO, which requires an 
SMC-dependent (but process independent) contribution which is very laborious to obtain (this is the reason why so far MC@NLO has been matched only to HERWIG). On the other hand, the technique of ref. [1] requires the use of a soft shower (the vetoed-truncated shower) which is not available in present SMC generators, but whose effects are expected to be small. Furthermore, the approach of ref. [1] generates events with positive weights, while MC@NLO generates also events with negative weight. For this reason the method has been referred to as POWHEG, for POsitive Weight Hard Event Generator. In ref. [6] the POWHEG method was successfully employed in the calculation of $Z$ pair production in hadronic collisions.

In the present work, we apply the POWHEG method to heavy flavour hadroproduction. NLO cross section formulae for this process have been available for a long time $[7,8,9]$. In the application of the POWHEG method, no new analytic results are needed. Furthermore, since the kinematics of the process is similar to that of $Z$ pair production [6], many results of that paper directly extend to the case at hand. The result of our work provides a valuable alternative to the MC@NLO program. It can be used with both HERWIG and PYTHIA [10], and it should not be difficult to interface it to other SMC's as well. It furthermore provides a viable alternative, in cases when negatively weighted events in MC@NLO cause severe problems, like, for example, when the heavy quark is relatively light compared to the energies involved.

The web location of the code repository can be found in the manual, ref. [11].

\section{Kinematics and cross section}

The differential cross section for the production of heavy quark pairs in hadronic collisions was computed in ref. [7], [8] and [9] up to order $\alpha_{s}^{3}$. In this section, we formulate the result of ref. [9] in a form which is suitable for the generation of the hardest emission using the procedure proposed in ref. [1].

The order- $\alpha_{s}^{3}$ cross section for the process $H_{1} H_{2} \rightarrow Q \bar{Q}+X$ can be written as the sum of four terms:

$$
d \sigma=d \sigma^{(\mathrm{b})}+d \sigma^{(\mathrm{sv})}+d \sigma^{(\mathrm{f})}+d \sigma^{(\mathrm{c})} .
$$

Here $d \sigma^{(\mathrm{b})}$ is the leading-order (Born) cross section. The term $d \sigma^{(\mathrm{sv})}$ collects order- $\alpha_{s}^{3}$ contributions with the same two-body kinematics as the Born term, namely one-loop corrections and real-emission contributions in the soft limit. Finally, $d \sigma^{(\mathrm{f})}$ represents the cross section for real emission, and the corresponding subtractions, in a generic configuration, while $d \sigma^{(\mathrm{c})}$ is a remnant of the subtraction of initial-state collinear singularities.

\subsection{Born and Soft-Virtual kinematics}

At leading order, the relevant parton subprocesses are

$$
\begin{aligned}
& g\left(p_{1}\right)+g\left(p_{2}\right) \rightarrow Q\left(k_{1}\right)+\bar{Q}\left(k_{2}\right), \\
& q\left(p_{1}\right)+\bar{q}\left(p_{2}\right) \rightarrow Q\left(k_{1}\right)+\bar{Q}\left(k_{2}\right),
\end{aligned}
$$


where $q$ is a quark or antiquark of any flavour, $\bar{q}$ the corresponding antiparticle, and $g$ is a gluon. Particle four-momenta are displayed in brackets; we have $p_{1}^{2}=p_{2}^{2}=0$, $k_{1}^{2}=k_{2}^{2}=m_{Q}^{2}$, where $m_{Q}$ is the heavy quark mass.

The kinematics of heavy quark pair production is entirely analogous to that of vector boson pair production. We introduce the reduced Mandelstam invariants ${ }^{1}$

$$
s=\left(p_{1}+p_{2}\right)^{2}, \quad t=\left(p_{1}-k_{1}\right)^{2}-m_{Q}^{2}, \quad u=\left(p_{1}-k_{2}\right)^{2}-m_{Q}^{2},
$$

related by $s+t+u=0$.

Event generation is conveniently performed in terms of the invariant mass $M_{Q \bar{Q}}$ and the rapidity $Y_{Q \bar{Q}}$ of the $Q \bar{Q}$ pair in the laboratory frame. They are given by

$$
\begin{aligned}
& M_{Q \bar{Q}}^{2}=\left(k_{1}+k_{2}\right)^{2}=x_{1} x_{2} S \\
& Y_{Q \bar{Q}}=\frac{1}{2} \log \frac{\left(p_{1}+p_{2}\right)^{0}+\left(p_{1}+p_{2}\right)^{3}}{\left(p_{1}+p_{2}\right)^{0}-\left(p_{1}+p_{2}\right)^{3}}=\frac{1}{2} \log \frac{x_{1}}{x_{2}},
\end{aligned}
$$

where $S$ is the squared center-of-mass energy of the colliding hadrons, and $x_{1}, x_{2}$ are the fractions of longitudinal momenta carried by the incoming partons. Equations $(2.4,2.5)$ yield

$$
x_{1}=\sqrt{\frac{M_{Q \bar{Q}}^{2}}{S}} e^{Y_{Q \bar{Q}}} \equiv x_{b 1} ; \quad x_{2}=\sqrt{\frac{M_{Q \bar{Q}}^{2}}{S}} e^{-Y_{Q \bar{Q}}} \equiv x_{b 2} ; \quad d x_{1} d x_{2}=\frac{1}{S} d Y_{Q \bar{Q}} d M_{Q \bar{Q}}^{2} .
$$

We adopt as two-body kinematic variables the set $v=\left\{M_{Q \bar{Q}}, Y_{Q \bar{Q}}, \cos \theta_{1}\right\}$ (which we will call the Born variables henceforth), where $\theta_{1}$ is the angle between $\vec{p}_{1}$ and $\vec{k}_{1}$ in the partonic center-of-mass frame, so that

$$
t=-\frac{M_{Q \bar{Q}}^{2}}{2}\left(1-\beta \cos \theta_{1}\right)
$$

where

$$
\beta=\sqrt{1-\rho} ; \quad \rho=\frac{4 m_{Q}^{2}}{M_{Q \bar{Q}}^{2}} .
$$

Using eq. (2.6) and the usual expression of the two-body phase space measure $d \Phi_{2}$, one immediately finds

$$
d \Phi_{2} d x_{1} d x_{2}=\frac{\beta}{16 \pi S} d \cos \theta_{1} d M_{Q \bar{Q}}^{2} d Y_{Q \bar{Q}} .
$$

In order to keep our notation similar to that of ref. [1], we define

$$
d \Phi_{v}=d \cos \theta_{1} d M_{Q \bar{Q}}^{2} d Y_{Q \bar{Q}}
$$

The appropriate integration region for the variables $v$ is

$$
4 m_{Q}^{2} \leq M_{Q \bar{Q}}^{2} \leq S, \quad \frac{1}{2} \log \frac{M_{Q \bar{Q}}^{2}}{S} \leq Y_{Q \bar{Q}} \leq-\frac{1}{2} \log \frac{M_{Q \bar{Q}}^{2}}{S}, \quad-1 \leq \cos \theta_{1} \leq 1 .
$$

\footnotetext{
${ }^{1}$ We depart slightly from the notation of ref. [6], where $t$ and $u$ have different definitions.
} 
The Born cross section is given by

$$
d \sigma^{(\mathrm{b})}=d \Phi_{v} \sum_{j} B_{j}(v, \mu)
$$

where

$$
B_{j}(v, \mu)=\frac{\beta}{16 \pi S} f_{j}^{H_{1}}\left(x_{b 1}, \mu\right) f_{-j}^{H_{2}}\left(x_{b 2}, \mu\right) \mathcal{M}_{j,-j}^{(\mathrm{b})}\left(M_{Q \bar{Q}}^{2}, t\right) .
$$

The index $j$ represents the light quarks and antiquarks $(j \neq 0)$ and the gluon $(j=0)$. It ranges between $-n_{\ell}$ and $n_{\ell}$, where $n_{\ell}$ is the number of light flavours. In the following we will also use $q$ to represent all values of $j$ except for $j=0, \bar{q}$ to represent $-j$, and $g$ to represent $j=0 . f_{j}^{H}(x, \mu)$ denotes the distribution function of parton $j$ in the hadron $H$, and $\mu$ is a factorization scale. The function $\mathcal{M}_{j,-j}^{(\mathrm{b})}(s, t)$ is the squared invariant amplitude, summed over final-state polarizations and averaged over initial-state polarizations and colours, divided by the relevant flux factor. We have

$$
\begin{aligned}
& \mathcal{M}_{g g}^{(\mathrm{b})}(s, t)=\frac{g^{4}}{2 s} \frac{2 T_{\mathrm{F}}}{D_{\mathrm{A}}}\left(C_{\mathrm{F}} \frac{s}{u t}-C_{\mathrm{A}}\right)\left(\frac{t^{2}}{s^{2}}+\frac{u^{2}}{s^{2}}+\rho-\frac{m_{Q}^{4}}{t u}\right), \\
& \mathcal{M}_{q \bar{q}}^{(\mathrm{b})}(s, t)=\frac{g^{4}}{2 s} \frac{C_{\mathrm{F}} T_{\mathrm{F}}}{N_{c}}\left(\frac{2 t^{2}}{s^{2}}+\frac{2 u^{2}}{s^{2}}+\rho\right),
\end{aligned}
$$

where $N_{c}=3, T_{\mathrm{F}}=1 / 2, C_{\mathrm{F}}=4 / 3, D_{\mathrm{A}}=8$.

Order- $\alpha_{s}^{3}$ contributions to the cross section arise from one-loop corrections to the twobody process eq. (2.2), and from real-emission subprocesses at tree level. The contribution of one-loop diagrams must be summed to the one-gluon emission cross section in the soft limit, in order to obtain an infrared-finite result. The resulting contribution, usually called the soft-virtual contribution, has the same kinematic structure as the leading-order term:

$$
d \sigma^{(\mathrm{sv})}=d \Phi_{v} \sum_{j} V_{j}(v, \mu)
$$

where

$$
V_{j}(v, \mu)=\frac{\beta}{16 \pi S} f_{j}^{H_{1}}\left(x_{b 1}, \mu\right) f_{-j}^{H_{2}}\left(x_{b 2}, \mu\right) \mathcal{M}_{j,-j}^{(\mathrm{sv})}\left(M_{Q \bar{Q}}^{2}, t, \mu^{2}\right) .
$$

The invariant amplitude $\mathcal{M}_{j,-j}^{(\mathrm{sv})}\left(M_{Q \bar{Q}}^{2}, t, \mu^{2}\right)$ is the sum of the virtual corrections to the 2 body subprocesses, and the soft contribution of real emission. The sum is finite, and it was computed in ref. [9].

\subsection{Real emission kinematics}

We consider now the real-emission subprocesses

$$
\begin{aligned}
& q\left(p_{1}\right)+\bar{q}\left(p_{2}\right) \rightarrow Q\left(k_{1}\right)+\bar{Q}\left(k_{2}\right)+g(k) \\
& q\left(p_{1}\right)+g\left(p_{2}\right) \rightarrow Q\left(k_{1}\right)+\bar{Q}\left(k_{2}\right)+q(k) \\
& g\left(p_{1}\right)+\bar{q}\left(p_{2}\right) \rightarrow Q\left(k_{1}\right)+\bar{Q}\left(k_{2}\right)+\bar{q}(k)
\end{aligned}
$$


in a generic kinematical configuration. The processes (2.17-2.19) are characterized by five independent scalar quantities, which we choose to be

$$
\begin{aligned}
& s=\left(p_{1}+p_{2}\right)^{2}, \quad t_{k}=\left(p_{1}-k\right)^{2}, \quad u_{k}=\left(p_{2}-k\right)^{2}, \\
& q_{1}=\left(p_{1}-k_{1}\right)^{2}-m_{Q}^{2}, \quad q_{2}=\left(p_{2}-k_{2}\right)^{2}-m_{Q}^{2},
\end{aligned}
$$

as in ref. [9]. We introduce the variables

$$
x=\frac{M_{Q \bar{Q}}^{2}}{s} ; \quad y=\cos \theta,
$$

where $\theta$ is the scattering angle of the emitted parton in the partonic center-of-mass system. With these definitions,

$$
t_{k}=-\frac{s}{2}(1-x)(1-y) ; \quad u_{k}=-\frac{s}{2}(1-x)(1+y) .
$$

It is easy to show that in the case of the subprocesses (2.17-2.19) one has

$$
Y_{Q \bar{Q}}=\frac{1}{2} \log \left[\frac{x_{1}}{x_{2}} \frac{s+u_{k}}{s+t_{k}}\right]=\frac{1}{2} \log \left[\frac{x_{1}}{x_{2}} \frac{2-(1-x)(1+y)}{2-(1-x)(1-y)}\right] ; \quad M_{Q \bar{Q}}^{2}=x x_{1} x_{2} S,
$$

and therefore

$$
x_{1}=\frac{x_{b 1}}{\sqrt{x}} \sqrt{\frac{2-(1-x)(1-y)}{2-(1-x)(1+y)}} ; \quad x_{2}=\frac{x_{b 2}}{\sqrt{x}} \sqrt{\frac{2-(1-x)(1+y)}{2-(1-x)(1-y)}}
$$

and

$$
d x_{1} d x_{2}=\frac{1}{x S} d M_{Q \bar{Q}}^{2} d Y_{Q \bar{Q}} .
$$

The range for the variable $x$ is restricted by the requirement that both $x_{1}$ and $x_{2}$ be smaller than one; this gives

$$
x_{\min } \leq x \leq 1,
$$

with

$$
\begin{aligned}
x_{\min }=\max & \left(\frac{2(1+y) x_{b 1}^{2}}{\sqrt{\left(1+x_{b 1}^{2}\right)^{2}(1-y)^{2}+16 y x_{b 1}^{2}}+(1-y)\left(1-x_{b 1}^{2}\right)},\right. \\
& \left.\frac{2(1-y) x_{b 2}^{2}}{\sqrt{\left(1+x_{b 2}^{2}\right)^{2}(1+y)^{2}-16 y x_{b 2}^{2}}+(1+y)\left(1-x_{b 2}^{2}\right)}\right) .
\end{aligned}
$$

Note that $x_{\min }$ depends explicitly on $y$, and implicitly on $M_{Q \bar{Q}}^{2}$ and $Y_{Q \bar{Q}}$ through $x_{b 1}, x_{b 2}$. It can be checked that $x_{\min }$ is always larger that $M_{Q \bar{Q}}^{2} / S$, as required by the definition of $x$.

In the center-of-mass frame of the $Q \bar{Q}$ system, the four-momenta of the produced heavy quarks can be parametrized in terms of two angles $\theta_{1}, \theta_{2}$ :

$$
\begin{aligned}
& k_{1}=\frac{M_{Q \bar{Q}}}{2}\left(1, \beta \sin \theta_{2} \sin \theta_{1}, \beta \cos \theta_{2} \sin \theta_{1}, \beta \cos \theta_{1}\right) \\
& k_{2}=\frac{M_{Q \bar{Q}}}{2}\left(1,-\beta \sin \theta_{2} \sin \theta_{1},-\beta \cos \theta_{2} \sin \theta_{1},-\beta \cos \theta_{1}\right),
\end{aligned}
$$


with $\beta$ given in eq. (2.8). Both $\theta_{1}$ and $\theta_{2}$ range between 0 and $\pi$. Thus, in addition to the Born variables $v$, we have now the three radiation variables $r=\left\{x, y, \theta_{2}\right\}$, with

$$
x_{\min } \leq x \leq 1, \quad-1 \leq y \leq 1, \quad 0 \leq \theta_{2} \leq \pi .
$$

Following ref. [1] we define the corresponding integration measure

$$
d \Phi_{r}=d x d y d \theta_{2} .
$$

From the computation of ref. [9] we obtain

$$
d \sigma^{(\mathrm{f})}=d \Phi_{v} d \Phi_{r}\left\{R_{g g}(v, r, \mu)+\sum_{q}\left[R_{q \bar{q}}(v, r, \mu)+R_{q g}(v, r, \mu)+R_{g \bar{q}}(v, r, \mu)\right]\right\}
$$

where

$$
\begin{aligned}
R_{g g}(v, r, \mu)= & \frac{1}{(4 \pi)^{2}} \frac{\beta}{64 \pi^{2} M_{Q \bar{Q}}^{2} S}\left(\frac{1}{1-x}\right)_{\rho}\left[\left(\frac{1}{1-y}\right)_{+}+\left(\frac{1}{1+y}\right)_{+}\right] \\
& f_{g}^{H_{1}}\left(x_{1}, \mu\right) f_{g}^{H_{2}}\left(x_{2}, \mu\right) f_{g g}\left(x, y, \theta_{1}, \theta_{2}, \mu\right) \\
R_{q \bar{q}}(v, r, \mu)= & \frac{1}{(4 \pi)^{2}} \frac{\beta}{64 \pi^{2} M_{Q \bar{Q}}^{2} S}\left(\frac{1}{1-x}\right)_{\rho}\left[\left(\frac{1}{1-y}\right)_{+}+\left(\frac{1}{1+y}\right)_{+}\right] \\
& f_{q}^{H_{1}}\left(x_{1}, \mu\right) f_{\bar{q}}^{H_{2}}\left(x_{2}, \mu\right) f_{q \bar{q}}\left(x, y, \theta_{1}, \theta_{2}, \mu\right) \\
R_{q g}^{ \pm}(v, r, \mu)= & \frac{1}{(4 \pi)^{2}} \frac{\beta}{64 \pi^{2} M_{Q \bar{Q}}^{2} S}\left(\frac{1}{1-x}\right)\left(\frac{1}{1 \mp y}\right)_{+} f_{q}^{H_{1}}\left(x_{1}, \mu\right) f_{g}^{H_{2}}\left(x_{2}, \mu\right) f_{q g}\left(x, y, \theta_{1}, \theta_{2}, \mu\right) \\
R_{g \bar{q}}^{ \pm}(v, r, \mu)= & \frac{1}{(4 \pi)^{2}} \frac{\beta}{64 \pi^{2} M_{Q \bar{Q}}^{2} S}\left(\frac{1}{1-x}\right)\left(\frac{1}{1 \mp y}\right)_{+} f_{g}^{H_{1}}\left(x_{1}, \mu\right) f_{\bar{q}}^{H_{2}}\left(x_{2}, \mu\right) f_{g \bar{q}}\left(x, y, \theta_{1}, \theta_{2}, \mu\right) .
\end{aligned}
$$

The functions $R_{q \bar{q}}, R_{q g}, R_{g \bar{q}}$ denote the regularized real emission cross sections for the different subprocesses. The functions $f_{q \bar{q}}$ and $f_{q g}$ are regular in the limits of soft $(x=1)$ or collinear $(y= \pm 1)$ emission; they are defined as in eq. (3.3) of ref. [9]. The distributions $1 /(1-x)_{\rho}$ and $1 /(1 \pm y)_{+}$are defined by

$$
\begin{aligned}
& \int_{\rho}^{1} d x g(x)\left(\frac{1}{1-x}\right)_{\rho}=\int_{\rho}^{1} d x \frac{g(x)-g(1)}{1-x} \\
& \int_{-1}^{1} d y h(y)\left(\frac{1}{1 \pm y}\right)_{+}=\int_{-1}^{1} d y \frac{h(y)-h(\mp 1)}{1 \pm y} .
\end{aligned}
$$

\subsection{Collinear remnants}

The remnants of the collinear subtraction must also be added to get the full cross section. This contribution has the form [9]

$$
\begin{aligned}
d \sigma^{(\mathrm{c})}= & d \Phi_{v} d x d y \\
& \left\{\left[L_{g g}^{+}(v, x, \mu)+\sum_{q}\left(L_{q \bar{q}}^{+}(v, x, \mu)+L_{g \bar{q}}^{+}(v, x, \mu)+L_{q g}^{+}(v, x, \mu)\right)\right] \delta(1-y)\right. \\
+ & {\left.\left[L_{g g}^{-}(v, x, \mu)+\sum_{q}\left(L_{q \bar{q}}^{-}(v, x, \mu)+L_{g \bar{q}}^{-}(v, x, \mu)+L_{q g}^{-}(v, x, \mu)\right)\right] \delta(1+y)\right\}, }
\end{aligned}
$$


where

$$
\begin{aligned}
L_{i j}^{+}(x)= & \frac{\alpha_{s}}{2 \pi} \frac{\beta}{16 \pi S} \sum_{i^{\prime}}\left\{\left[(1-x) P_{i i^{\prime}}(x, 0)\right]\left[\left(\frac{1}{1-x}\right)_{\rho} \log \frac{M_{Q \bar{Q}}^{2}}{x \mu^{2}}+2\left(\frac{\log (1-x)}{1-x}\right)_{\rho}\right]\right. \\
& \left.-\left[(1-x) P_{i i^{\prime}}^{\prime}(x, 0)\right]\left(\frac{1}{1-x}\right)_{\rho}\right\} \mathcal{M}_{i^{\prime} j}^{(\mathrm{b})}\left(M_{Q \bar{Q}}^{2}, t\right) f_{i}^{H_{1}}\left(x_{1}, \mu\right) f_{j}^{H_{2}}\left(x_{2}, \mu\right)
\end{aligned}
$$

and

$$
\begin{aligned}
L_{i j}^{-}(x)= & \frac{\alpha_{s}}{2 \pi} \frac{\beta}{16 \pi S} \sum_{j^{\prime}}\left\{\left[(1-x) P_{j j^{\prime}}(x, 0)\right]\left[\left(\frac{1}{1-x}\right)_{\rho} \log \frac{M_{Q \bar{Q}}^{2}}{x \mu^{2}}+2\left(\frac{\log (1-x)}{1-x}\right)_{\rho}\right]\right. \\
& \left.-\left[(1-x) P_{j j^{\prime}}^{\prime}(x, 0)\right]\left(\frac{1}{1-x}\right)_{\rho}\right\} \mathcal{M}_{i j^{\prime}}^{(\mathrm{b})}\left(M_{Q \bar{Q}}^{2}, t\right) f_{i}^{H_{1}}\left(x_{1}, \mu\right) f_{j}^{H_{2}}\left(x_{2}, \mu\right)
\end{aligned}
$$

where $P_{i j}(x, \epsilon)$ are the leading-order Altarelli-Parisi splitting functions in $d=4-2 \epsilon$ dimensions, $P_{i j}^{\prime}(x, \epsilon)$ their first derivatives with respect to $\epsilon$, and $t$ is given in eq. (2.7). From eq. (2.28) we see that the integration range becomes $x_{b 1}<x<1$ when $y=1$, and $x_{b 2}<x<1$ for $y=-1$.

\section{Cross section for the hardest emission}

The POWHEG method, when applied to a generic process, may require a separated treatment of each singular region. In the present case (as in the case of $Z$ pair production) this is not needed. Our choice of variables $v, r$ is adequate for both collinear regions at the same time, the only difference being the sign of $y$. We have instead to pay attention to the flavour structure of the process. In ordinary SMC codes, the flavour structure of the Born subprocess is not altered by subsequent radiation. On the other hand, if the hardest radiation is produced in the context of a NLO calculation, the association of the NLO process with a Born subprocess is not always obvious. A given real-emission contribution must be associated with its underlying Born process, i.e. the Born process in which it factorizes in the collinear limit. In the present case, the collinear regions for the real $q \bar{q}$ subprocess always factorize in terms of the $q \bar{q}$ underlying Born, and the collinear regions for the real $g g$ subprocess factorize in terms of the $g g$ Born process. For the $q g(g \bar{q})$ processes, there are instead two possibilities (see fig. 1): the underlying Born process is $g g(q \bar{q})$ for the $y=1$ collinear region, and $q \bar{q}(g g)$ for $y=-1$. This is the reason why in eq. (2.33) we have separated the two collinear contributions $R_{q g}^{ \pm}$and $R_{g \bar{q}}^{ \pm}$. Thus, for a given flavour $q$, we lump together the $q \bar{q}$, the $q g$ and the $g \bar{q}$ real-emission subprocesses. 

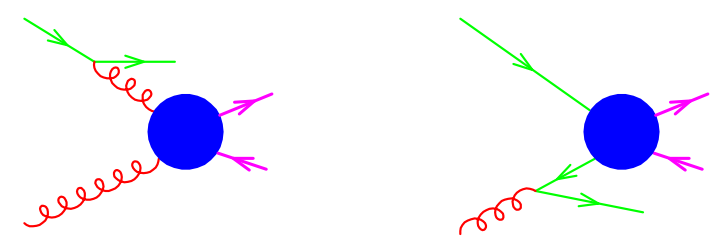

Figure 1: Different underlying Born structure in the two collinear regions of the $q g$ subprocess.

Following ref. [1], we write the cross section for the event with the hardest emission as

$$
\begin{aligned}
& d \sigma=\sum_{q} \bar{B}_{q}\left(v, \mu_{v}\right) d \Phi_{v}\left[\Delta_{q}(0)+\Delta_{q}\left(k_{\mathrm{T}}\right) \frac{\hat{R}_{q \bar{q}}\left(v, r, \mu_{r}\right)+\hat{R}_{q g}^{-}\left(v, r, \mu_{r}\right)+\hat{R}_{g \bar{q}}^{+}\left(v, r, \mu_{r}\right)}{B_{q}\left(v, \mu_{r}\right)} d \Phi_{r}\right] \\
& +\bar{B}_{g}\left(v, \mu_{v}\right) d \Phi_{v}\left[\Delta_{g}(0)+\Delta_{g}\left(k_{\mathrm{T}}\right) \frac{\hat{R}_{g g}\left(v, r, \mu_{r}\right)+\sum_{q}\left(\hat{R}_{q g}^{+}\left(v, r, \mu_{r}\right)+\hat{R}_{g \bar{q}}^{-}\left(v, r, \mu_{r}\right)\right)}{B_{q}\left(v, \mu_{r}\right)} d \Phi_{r}\right]
\end{aligned}
$$

where $\hat{R}_{i j}$ is obtained from $R_{i j}$ by removing the + prescriptions that regularize the $x$ and $y$ singularities. The $\hat{R}_{i j}$ are thus the unregularized real emission cross sections (corresponding to $R$ in the notation of ref. [1]). Notice that the real emission contributions having the same underlying Born configuration are grouped together in eq. (3.1). Furthermore,

$$
\begin{aligned}
\bar{B}_{q}(v, \mu)= & B_{q}(v, \mu)+V_{q}(v, \mu)+\int d \Phi_{r}\left[R_{q \bar{q}}(v, r, \mu)+R_{q g}^{-}(v, r, \mu)+R_{g \bar{q}}^{+}(v, r, \mu)\right] \\
& +\int_{-1}^{1} d y \int_{x_{\min }}^{1} d x\left[L_{q \bar{q}}^{+}(v, x, \mu)+L_{g \bar{q}}^{+}(v, x, \mu)\right] \delta(1-y) \\
& +\int_{-1}^{1} d y \int_{x_{\min }}^{1} d x\left[L_{q \bar{q}}^{-}(v, x, \mu)+L_{q g}^{-}(v, x, \mu)\right] \delta(1+y) \\
\bar{B}_{g}(v, \mu)= & B_{g}(v, \mu)+V_{g}(v, \mu)+\int d \Phi_{r}\left[R_{g g}(v, r, \mu)+\sum_{q}\left(R_{q g}^{+}(v, r, \mu)+R_{g \bar{q}}^{-}(v, r, \mu)\right)\right] \\
& +\int_{-1}^{1} d y \int_{x_{\min }}^{1} d x\left[L_{g g}^{+}(v, x, \mu)+\sum_{q} L_{q g}^{+}(v, x, \mu)\right] \delta(1-y) \\
& +\int_{-1}^{1} d y \int_{x_{\min }}^{1} d x\left[L_{q \bar{q}}^{-}(v, x, \mu)+\sum_{q} L_{g \bar{q}}^{-}(v, x, \mu)\right] \delta(1+y) \\
\Delta_{q}\left(p_{\mathrm{T}}\right)= & \exp \left[-\int^{\hat{R}_{q \bar{q}}\left(v, r, \mu_{r}\right)+\hat{R}_{q g}^{-}\left(v, r, \mu_{r}\right)+\hat{R}_{g \bar{q}}^{+}\left(v, r, \mu_{r}\right)} B_{q}\left(v, \mu_{r}\right)\right.
\end{aligned}
$$


$\Delta_{g}\left(p_{\mathrm{T}}\right)=\exp \left[-\int \frac{\hat{R}_{g g}\left(v, r, \mu_{r}\right)+\sum_{q} \hat{R}_{q g}^{+}\left(v, r, \mu_{r}\right)+\sum_{q} \hat{R}_{g \bar{q}}^{-}\left(v, r, \mu_{r}\right)}{B_{g}\left(v, \mu_{r}\right)} \theta\left(k_{\mathrm{T}}(v, r)-p_{\mathrm{T}}\right) d \Phi_{r}\right]$

and $k_{\mathrm{T}}(v, r)$ is the transverse momentum of the radiated parton,

$$
k_{\mathrm{T}}(v, r)=\sqrt{\frac{M_{Q \bar{Q}}^{2}}{4 x}(1-x)^{2}\left(1-y^{2}\right)} .
$$

Equation (3.1) is the analogue of eq. (5.10) of ref. [1]. The function $\Delta_{q}\left(p_{\mathrm{T}}\right)$ corresponds to $\Delta_{R}^{(\mathrm{NLO})}\left(p_{\mathrm{T}}\right)$ in the notation of ref. [1].

\section{Generation of the hardest event}

The generation of the hardest event according to eq. (3.1) can be performed in full analogy to the case of ref. [6]. We refer the reader to that paper for details. Here we only point out the relevant differences with respect to that case.

The generation of the Born configuration involves in this case two kinds of Born processes, the $q \bar{q}$ and $g g$ processes. The total cross section is thus given by

$$
\sigma_{\text {tot }}=\int d \Phi_{v}\left[\bar{B}_{g}\left(v, \mu_{v}\right)+\sum_{q} \bar{B}_{q}\left(v, \mu_{v}\right)\right] .
$$

After the Born configuration has been generated, one chooses the process (i.e. $g$ or a given flavour of $q$ ) with a probability proportional to $\bar{B}_{g}\left(v, \mu_{v}\right), \bar{B}_{q}\left(v, \mu_{v}\right)$. According to whether a $q$ or a $g$ was selected, one follows the same procedure of ref. [6] using to the first or second line of eq. (3.1) respectively.

\section{Accuracy of the Sudakov form factor}

Unlike the case of $Z$ pair production, in the case at hand the procedure illustrated in section 4 of ref. [6] (i.e. the redefinition of $\alpha_{s}$ given in eq. (4.9) of [6]) is not sufficient to guarantee full next-to-leading logarithmic accuracy of the Sudakov form factor. This is related to the fact that the heavy flavour production process at the Born level involves more than 3 coloured partons [12], so that soft emission cross sections do not simply factorize in terms of the Born cross section. Thus, the Sudakov form factor is strictly only accurate to leading log. In fact, next-to-leading logarithmic accuracy can be easily recovered at least in the large $N_{c}$ limit, where $N_{c}$ is the number of colours, with a procedure discussed in ref. [13]. The implementation of this procedure and the assessment of its impact is left for future work.

\section{Colour assignment}

In order to interface POWHEG with a shower Monte Carlo, colour connections must be specified. In the case of $Z$ pair production, only one colour structure is present. The 
situation is more complex in the case at hand, since more colour structures are relevant. This problem is dealt with in exactly the same way as was done in ref. [5], section 6.1. We used in fact the same decomposition of the large $N_{c}$ heavy flavour production cross section into contributions with different colour structure, and pick the colour structure with probability proportional to the value of the corresponding contribution.

\section{Results}

In this section, we present results obtained with the POWHEG method for a choice of observables relevant to heavy quark production. Our results will be compared to those obtained with MC@NLO. We thus interface POWHEG with HERWIG, in order to make a consistent comparison. The formalisms of POWHEG and MC@NLO differ in the treatment of contributions of orders higher than NLO, which are beyond the level of accuracy of the theoretical computations presently available. The difference is mainly due to the way the radiation of matrix element origin is generated, which is typically the hardest radiation. Furthermore, scale choices in the two codes are not the same. Therefore, sizable differences between the two methods are to be expected for bottom and charm production, where the relevant scale is relatively small, while in the case of top production the discrepancies should in principle be much less important. However, a detailed comparison between the two methods is beyond our present purposes, and it is left for future work.

We will consider two experimental configurations: $p \bar{p}$ collisions at $\sqrt{s}=1960 \mathrm{GeV}$, corresponding to the Tevatron Run II configuration, and $p p$ collisions at $\sqrt{s}=14 \mathrm{TeV}$, corresponding to the LHC. The results presented in this section have been obtained by setting the top and bottom masses equal to $172 \mathrm{GeV}$ and $4.75 \mathrm{GeV}$ respectively. We have used the MRST2002 [14] set of parton distributions. When considering the decay of top quarks, we have set $\Gamma_{t}=1.31 \mathrm{GeV}$.

We begin by considering top production. Both the POWHEG and MC@NLO codes include the possibility of generating distributions for either undecayed top quarks (which we will refer to as "stable top" in the following), or for the decay products of top quarks, taking spin correlations into account. We will show examples of both cases. We present in fig. 2 the single-inclusive transverse momentum distribution for a stable top quark produced at the Tevatron. The ratio between the POWHEG and the MC@NLO results is also shown in the lower pane. The POWHEG (solid histogram) and MC@NLO (dashed histogram) results are very close to each other over the whole range considered, except in the very small $p_{\mathrm{T}}$ region, where the POWHEG cross section tends to be larger.

In fig. 3 we present the distributions of the invariant mass and of the transverse momentum of a stable $t \bar{t}$ pair. The agreement in this case is also quite good; the POWHEG $p_{\mathrm{T}}(t \bar{t})$ distribution is slightly softer than that of MC@NLO.

The same observables are in an even better agreement in the case of the LHC. This is shown in figs. 4 and 5. This can be understood as a consequence of the fact that the kinematics of the production process is less constrained than at the Tevatron, therefore making the contribution of potentially large logarithms in the perturbative coefficients less important. 

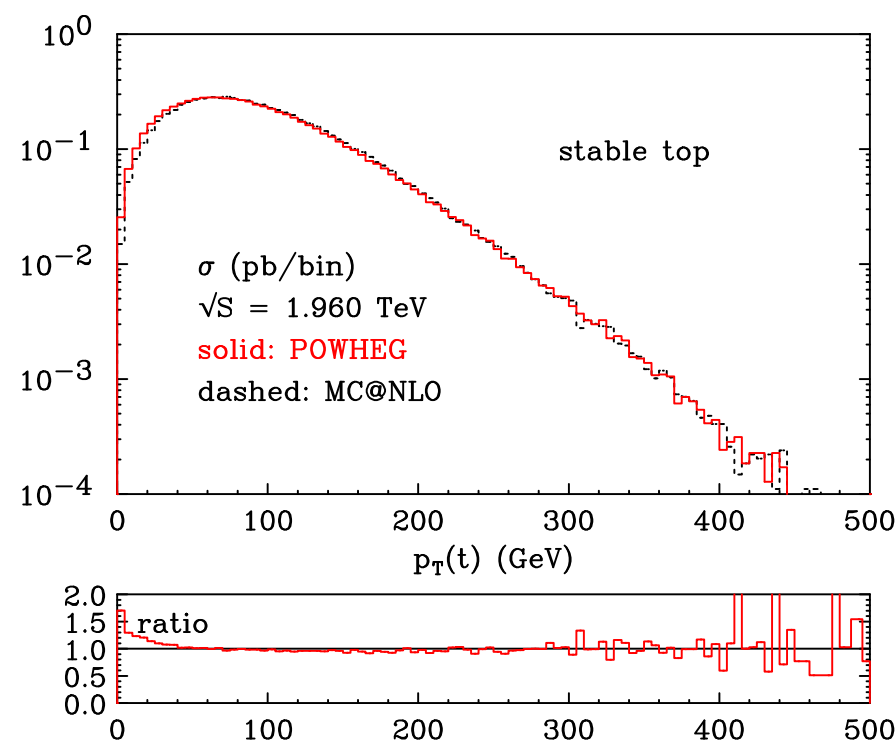

Figure 2: Transverse momentum distribution of a top quark at the Tevatron.
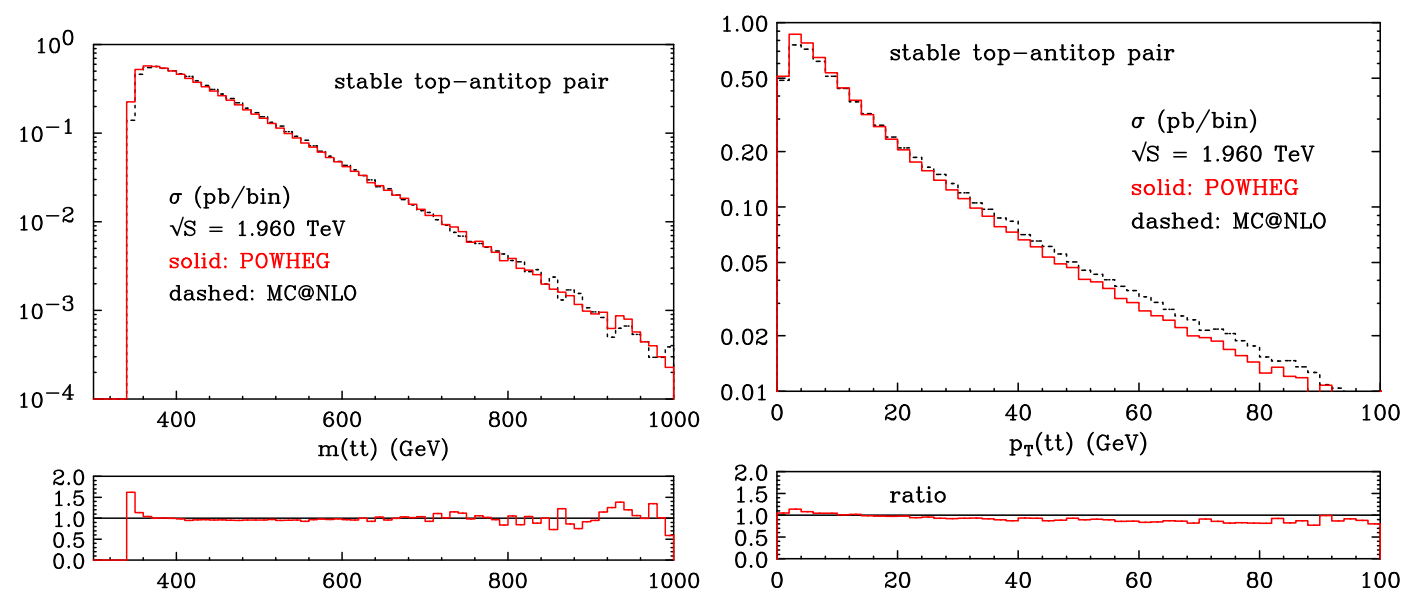

Figure 3: Invariant mass and transverse momentum distributions of $t \bar{t}$ pairs at the Tevatron.

We now turn to distributions of the decay products of unstable top quarks. We consider here only the dilepton channel, which considerably simplifies the analysis. In fig. 6 we show two representative single-inclusive distributions, namely the transverse momentum and the rapidity of negatively-charged lepton resulting from the $\bar{t}$ decay, in the Tevatron configuration. Again, both the POWHEG and the MC@NLO predictions are shown, together with the ratio between the two results. We see that no significant difference is present. Similar conclusions hold for the invariant mass and for the transverse momentum distributions of the charged-lepton pair, fig. 7, and for the azimuthal distance between the two charged leptons, fig. 8. A similarly good agreement is obtained for the same set of observables computed in the LHC configuration, figs. 9, 10, and 11 . The only visible 

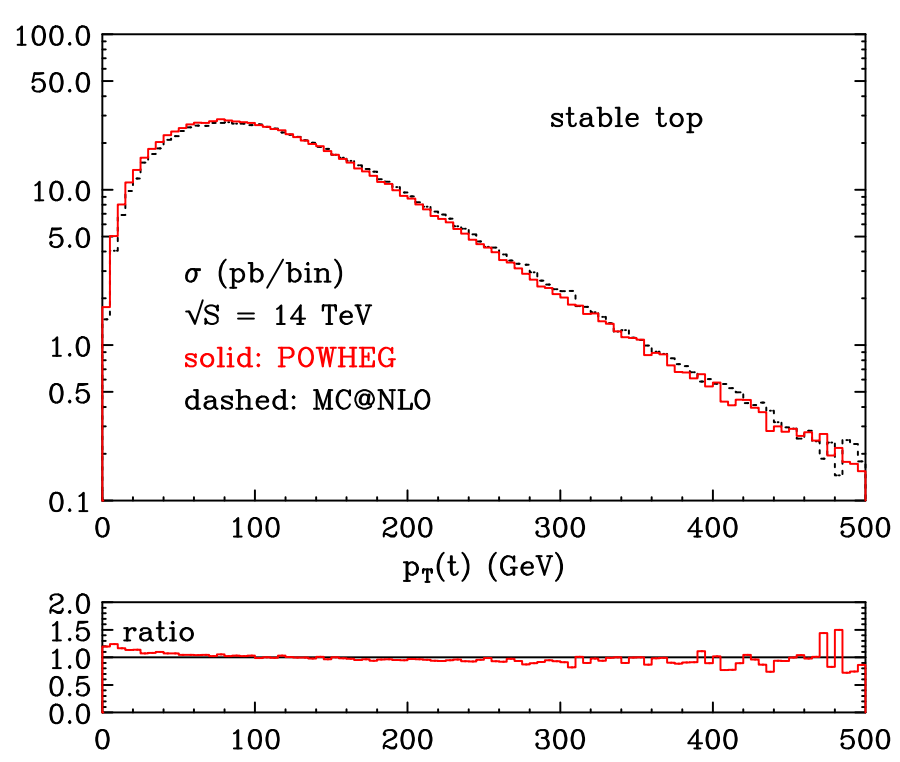

Figure 4: Transverse momentum distribution of a top quark at the LHC.
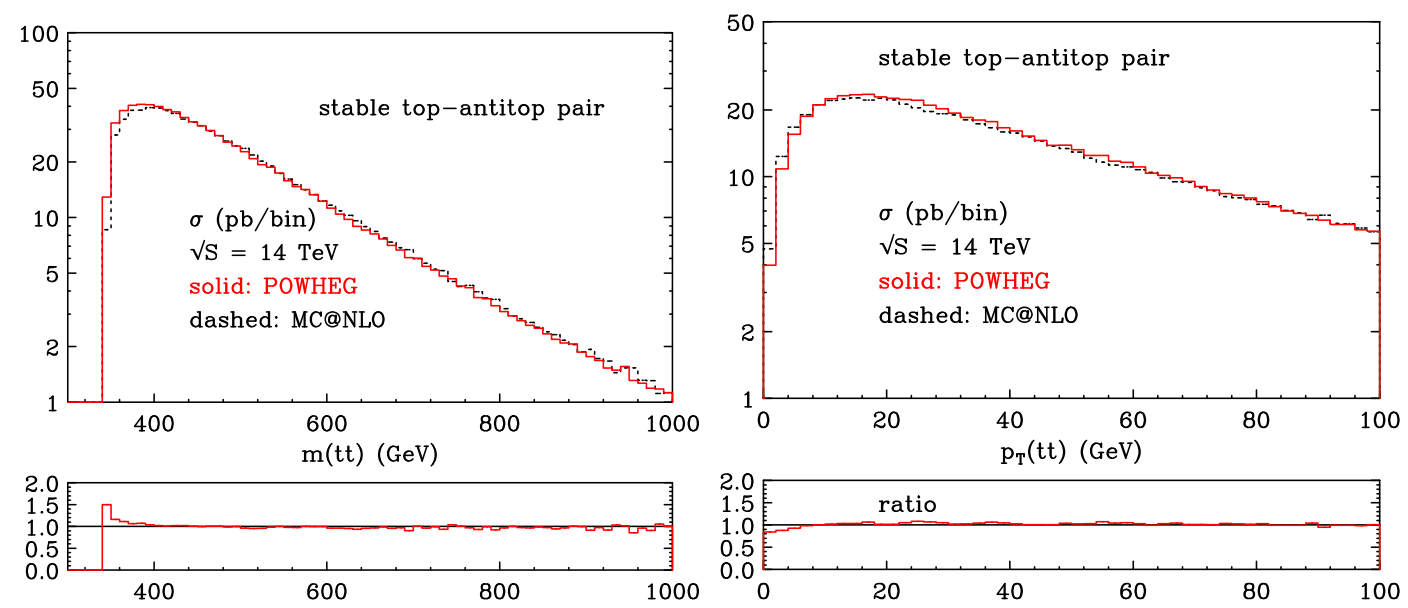

Figure 5: Invariant mass and transverse momentum distributions of $t \bar{t}$ pairs at the LHC.

difference is in the overall normalization, which is manifest in figs. 8 and 11 . This is due to the different choice of scales in the two computations.

We now turn to the case of bottom production. As is well known, perturbative NLO corrections to bottom production are very large, which implies that yet higher-order contributions are due to play a non-negligible role. As mentioned above, we therefore expect that POWHEG and MC@NLO will show larger discrepancies than in the case of top production purely on the basis of fixed-order expansion. There are, however, other sources of differences between the two approaches. Although both codes have been interfaced to HERWIG in order to obtain the results shown here, the logarithmically-enhanced terms beyond the leading one are not the same in the two approaches. Furthermore, if POWHEG 

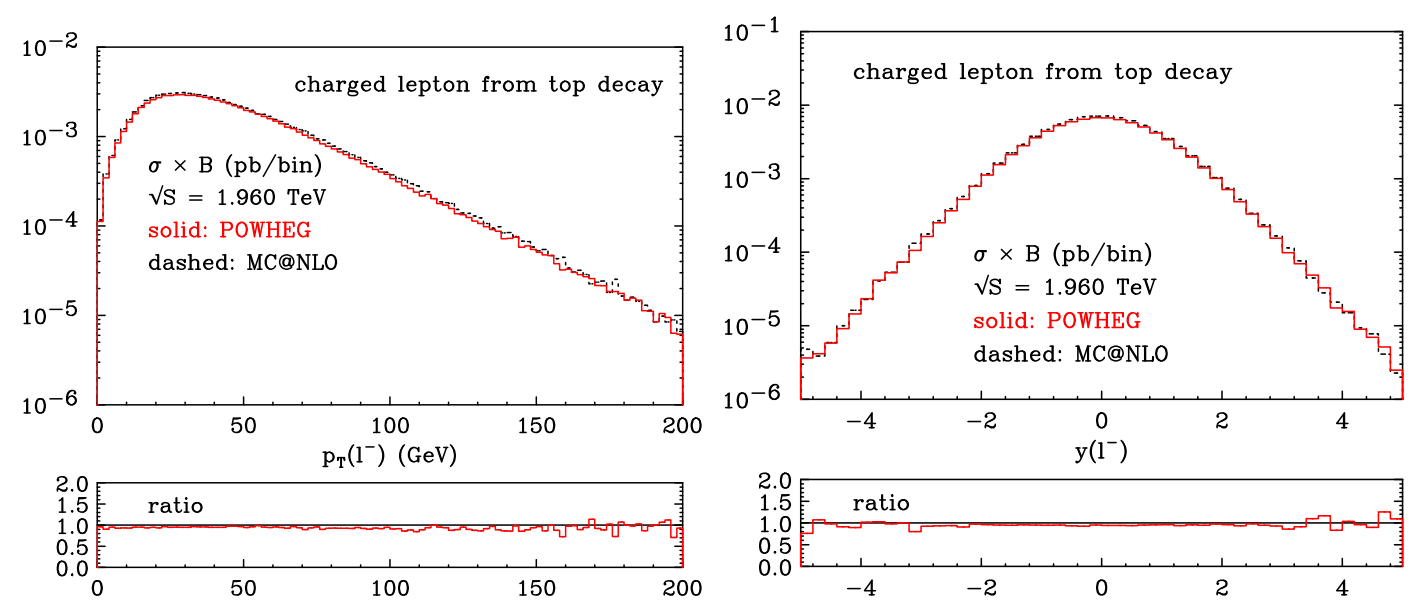

Figure 6: Transverse momentum and rapidity of a charged lepton from top decay at the Tevatron.
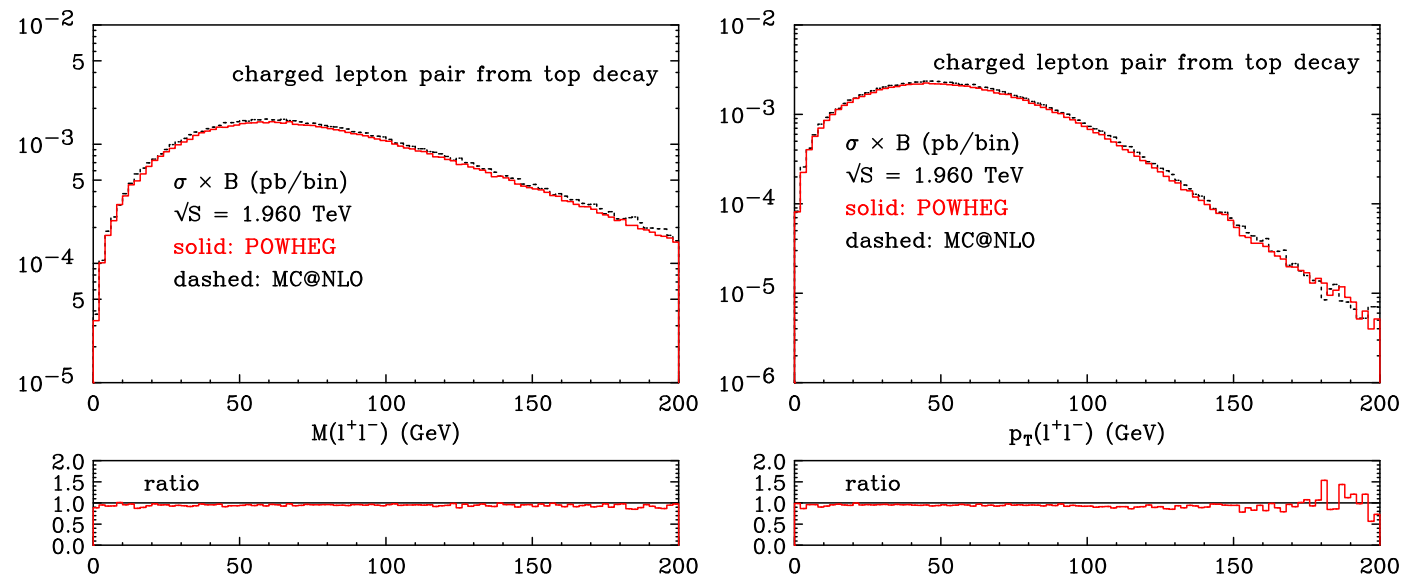

Figure 7: Invariant mass and transverse momentum distributions of $\ell^{+} \ell^{-}$pairs from top decay at the Tevatron.

is interfaced to an MC based on angular-ordered evolution (such as HERWIG), standard showers need be supplemented by truncated showers, whose effect is that of restoring colour coherence, which is lost because of the requirement that the hardest radiation be always the first. Since truncated showers are inherently soft, there are reasons to believe that their effects are not too large. At present, the only study of the impact of truncated showers has been performed in ref. [15]. There, a POWHEG implementation of $e^{+} e^{-}$annihilations into hadrons, interfaced to the HERWIG++ Monte Carlo [16], was presented. The effect of the truncated shower was found to be small. No studies have been performed in the case of hadron collisions.

In fig. 12 we present sample comparisons between POWHEG and MC@NLO results for bottom production at the Tevatron. All observables shown are relevant to lowest-lying $b$-flavoured meson states. We show the single-inclusive $p_{\mathrm{T}}$ (upper left pane), the pair $p_{\mathrm{T}}$ 

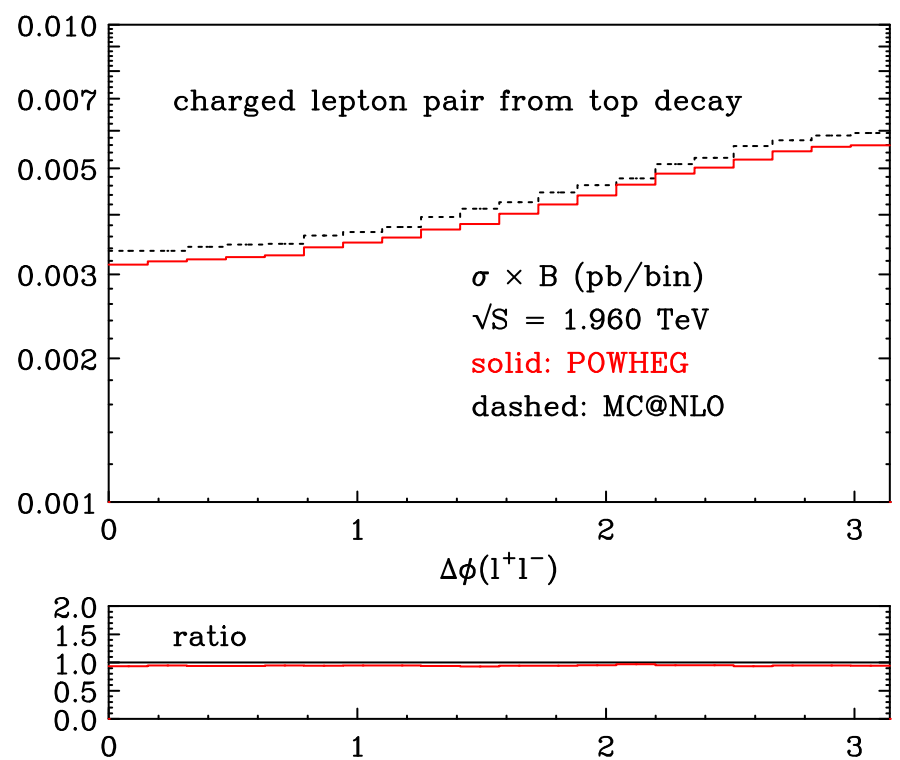

Figure 8: Distribution of the azimuthal distance between charged leptons from top decay at the Tevatron.
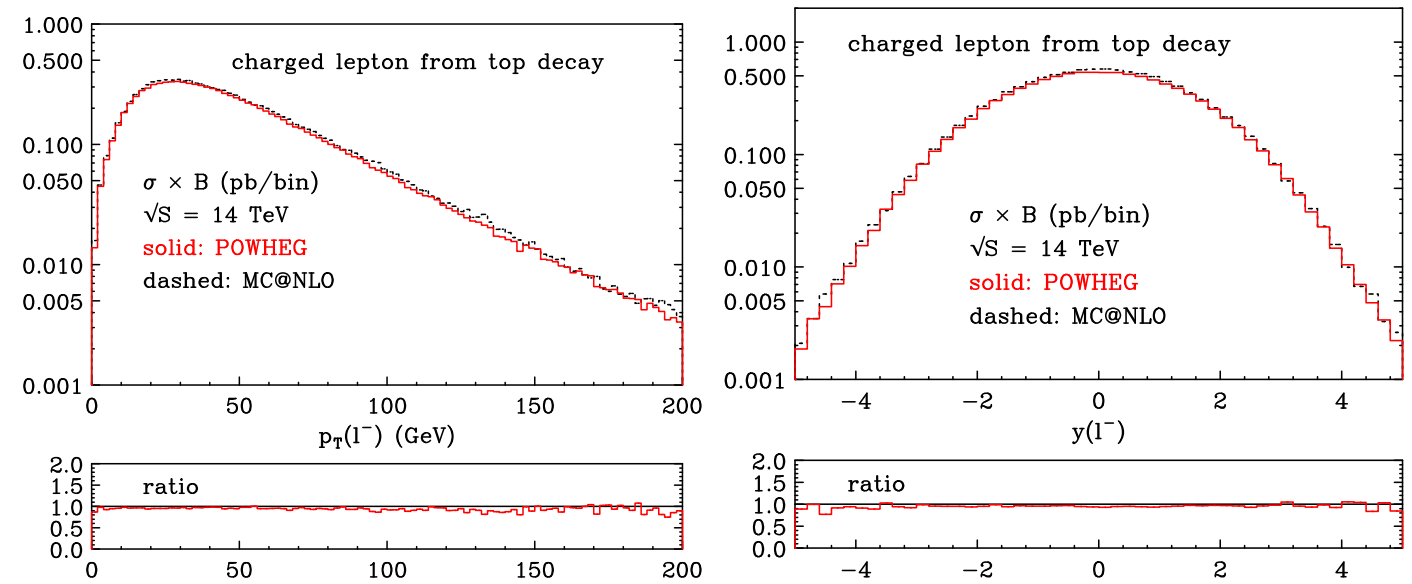

Figure 9: Transverse momentum and rapidity of a charged lepton from top decay at the LHC.

(upper right pane), and the azimuthal distance, without (lower left pane) and with (lower right pane) kinematic cuts; in the latter case, the cuts $|y|<1$ and $p_{\mathrm{T}}>5 \mathrm{GeV}$ are applied to both the $B$ 's of the pair. The two $p_{\mathrm{T}}$ distributions show a fair agreement, with POWHEG marginally (for single inclusive $p_{\mathrm{T}}$ ) or markedly (for the tail of the $p_{\mathrm{T}}$ of the pair) harder than MC@NLO. There are very clear differences in shape between the two azimuthal distributions. The discrepancy tends to be smaller when cuts are applied. As for the $p_{\mathrm{T}}$ of the pair, POWHEG gives harder results than MC@NLO, which we attribute mainly to the different treatment of hard radiation in the two formalisms. Finally, we point out that the POWHEG code is capable of producing bottom and charm distributions at the 

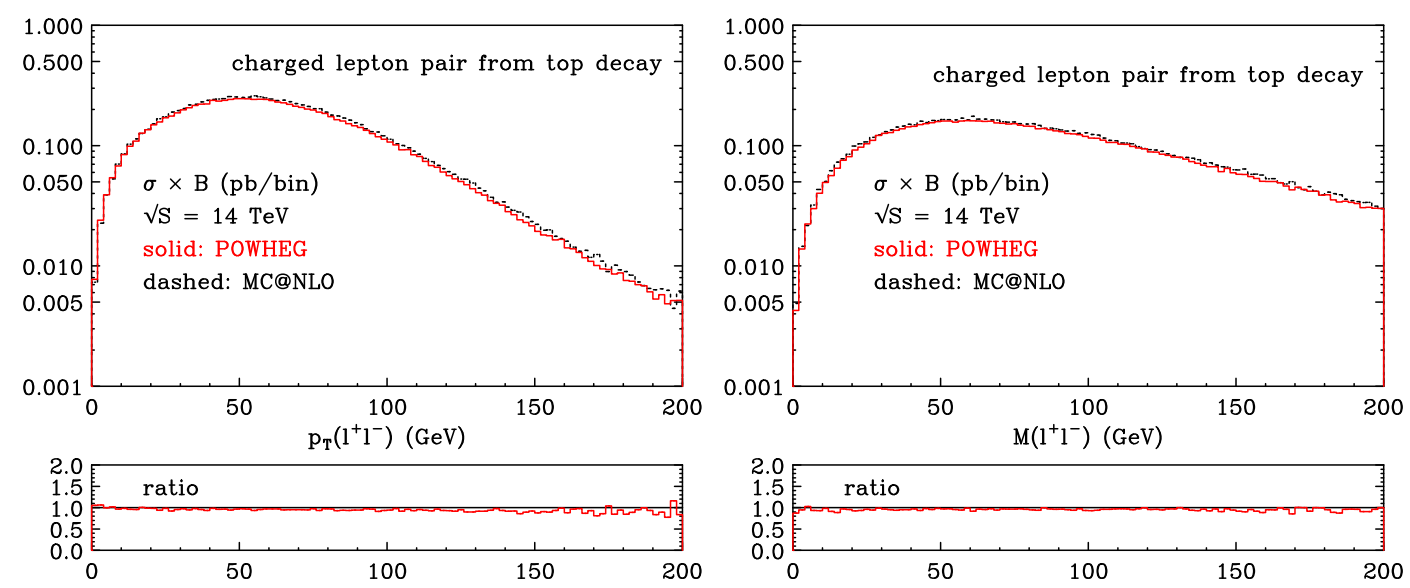

Figure 10: Invariant mass and transverse momentum distributions of $\ell^{+} \ell^{-}$pairs from top decay at the LHC.
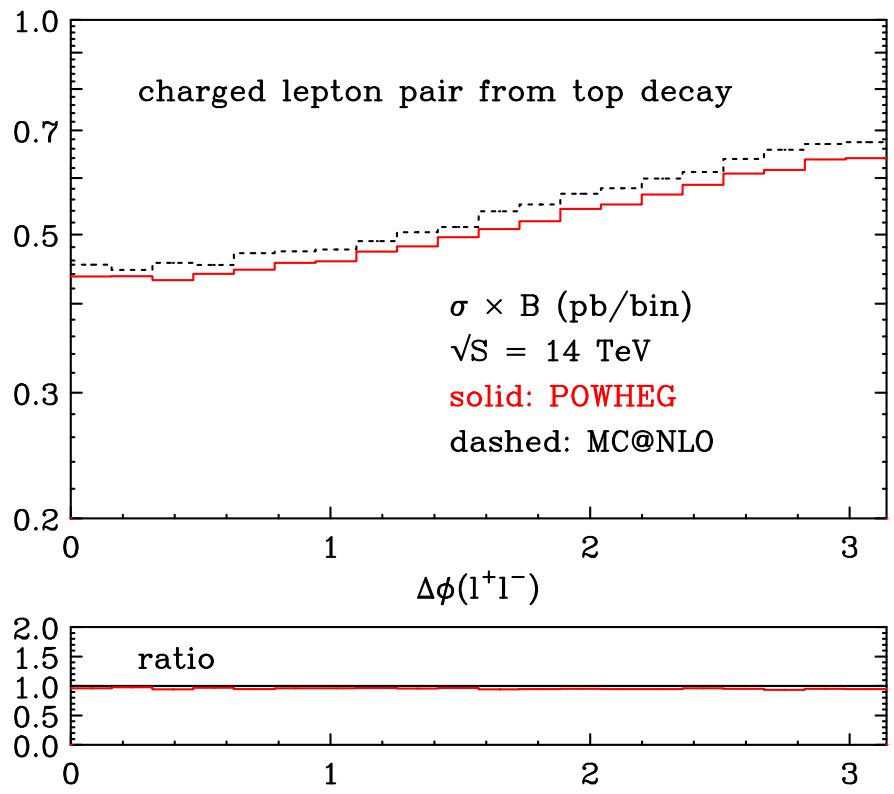

Figure 11: Distribution of the azimuthal distance between charged leptons from top decay at the LHC.

LHC energy, essentially without negatively-weighted events. In ref. [11] example input files are provided for bottom and charm production at the Tevatron and at the LHC, interfaced to both HERWIG and PYTHIA.

\section{Conclusions}

In this paper we have presented the implementation of heavy quark pair production according to the POWHEG formalism, which allows an NLO QCD computation to be matched 

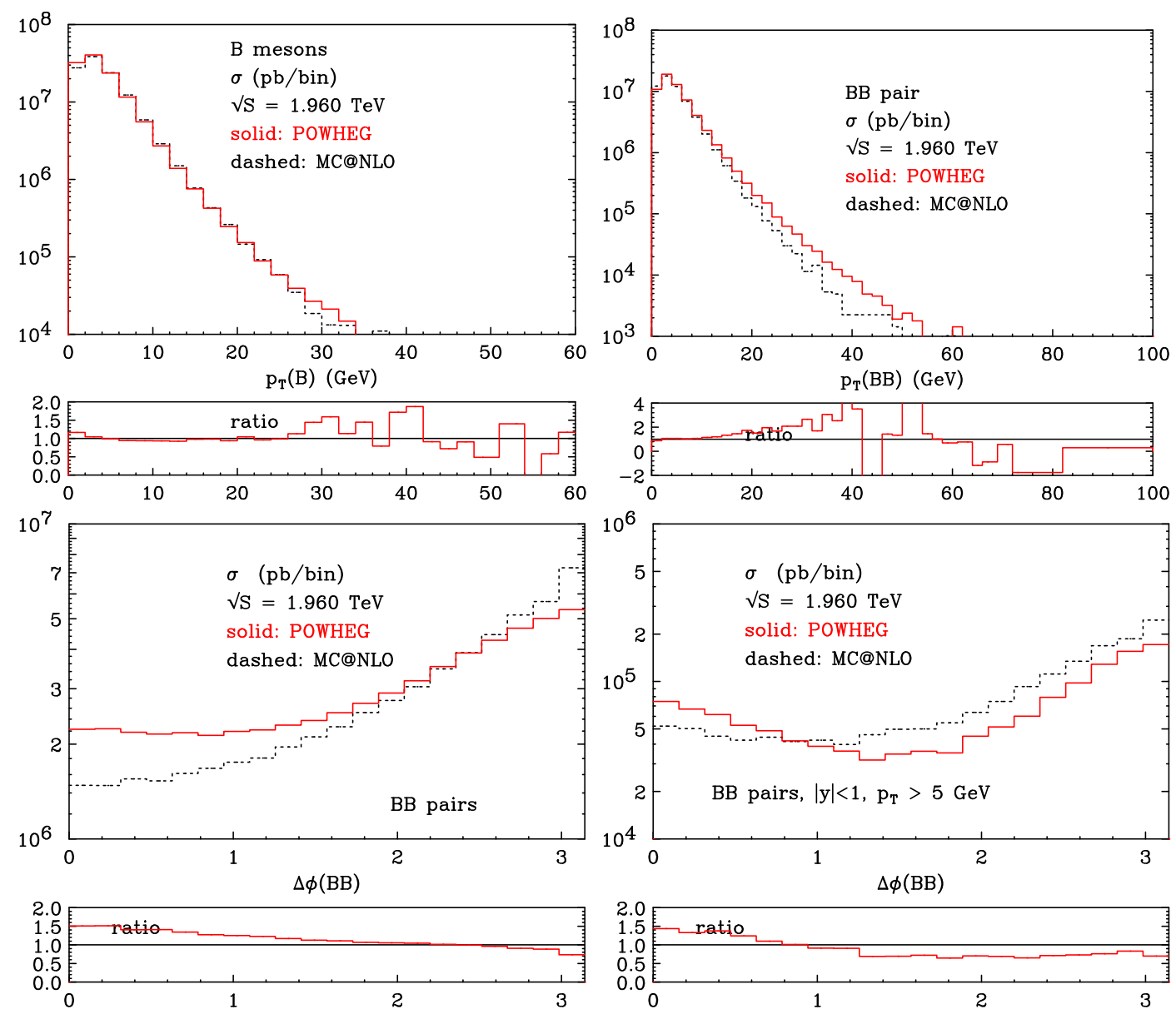

Figure 12: Bottom hadron distributions at the Tevatron.

with Parton Shower simulations. The fortran code we have constructed can be used to predict any infrared safe observable in $t \bar{t}, b \bar{b}$, and $c \bar{c}$ production at hadron colliders. We have compared our results with MC@NLO for $t \bar{t}$ production at the LHC and at the Tevatron, and for $b \bar{b}$ production at the Tevatron. In the case of top production, we observe a very good agreement between POWHEG and MC@NLO, for all the observables we have considered. On the other hand, the two approaches differ significantly for some observables in $b \bar{b}$ production, which implies that for such low-mass quarks perturbative corrections of order higher than next to leading are likely to play a non-negligible role.

In general, the agreement of the MC@NLO and POWHEG approaches is quite remarkable, in view of the fact that the two methods differ considerably in several aspects, summarized below:

1. The Sudakov form factors in MC@NLO and POWHEG are different: MC@NLO uses HERWIG's Sudakov form factor, POWHEG has its own (see eqs. (3.4) and (3.5)).

2. The hardest emission in POWHEG carries a strong coupling evaluated at the $p_{\mathrm{T}}$ of the emission, and a Sudakov form factor. On the other hand, in MC@NLO only $\mathbb{S}$ 
events, ${ }^{2}$ that have all emissions entirely performed by the shower, have these features. The $\mathbb{H}$ events are evaluated at the scale of the hard process, and they carry no Sudakov damping for small transverse momenta. This difference may show up for relatively low transverse momentum. We remind the reader that $\mathbb{H}$ events can have negative weight, so that it is difficult to understand in which direction this difference affects the results.

3. The POWHEG approach lacks the truncated showers.

4. Subleading terms in the shower may differ in the two approaches, due to the reshuffling of the splitting processes in the shower illustrated in ref. [1].

5. The $\mathbb{H}$ events in MC@NLO may be followed by radiation, generated by the shower, with a $p_{\mathrm{T}}$ harder than the $p_{\mathrm{T}}$ of the $\mathbb{H}$ event. In POWHEG harder emissions from the shower are always vetoed.

Because of the many differences, it is also difficult at this stage to understand what causes the differences in the distributions we have presented. Here we just make a few speculations about the possible origin of the differences, that should only be taken as hints for further studies. First, we look at top production. We see there that the inclusive $p_{\mathrm{T}}$ spectrum, the mass of the pair and the $p_{\mathrm{T}}$ of the pair differ in the very small $p_{\mathrm{T}}$ or $m(t t)$ region, POWHEG being generally higher (see figs. 2 to 5 ). The lack of soft-truncated showers in POWHEG (item 3 of the above list) could possibly cause this effect. On the other hand, soft radiation is also treated differently as far as the hardest emission is concerned, as specified in item 2 of the above list. The fact that the difference goes in the opposite way (i.e. POWHEG is below MC@NLO) for the transverse momentum of the top pair at LHC also shows that the lack of truncated showers (that would lower the POWHEG distribution) cannot be the whole answer. A second effect we notice is the considerable difference in the azimuthal distance of the bottom pair (see fig. 12) especially in the region where the two heavy mesons are near in azimuth. Here, the lack of soft-truncated showers in POWHEG, or differences in subleading shower effects in the two methods, could yield a different degree of smearing of the azimuthal distance. The POWHEG result seems to have more smearing than the MC@NLO result. The lack of soft-truncated showers is likely to have the opposite effect. Also, the faster rise for small azimuthal difference is unlikely to be due to shower effects in general, and would suggest to look for effects in the hard radiation mechanism. Thus, items 2, 4 and 5 may be responsible for these differences. The third effect we consider is the harder tail of the $p_{\mathrm{T}}$ distribution of bottom pairs in POWHEG. This can only be ascribed to genuine higher order effects in the hard emission, as may arise from items 1 and $2 .^{3}$

\footnotetext{
${ }^{2}$ See refs. [4, 5] for the definition of $\mathbb{S}$ and $\mathbb{H}$ events.

${ }^{3}$ In a yet unpublished revision of the MC@NLO code the $p_{\mathrm{T}}$ spectra for $B$ production turn out to be harder than the ones shown here. The azimuthal distance distributions do not differ from those presented here.
} 


\section{Acknowledgement}

We thank Rachid Guernane for testing our code and suggesting speed improvements.

\section{References}

[1] P. Nason, A new method for combining NLO QCD with Shower Monte Carlo algorithms, JHEP 11 (2004) 040, [hep-ph/0409146].

[2] G. Corcella et al., HERWIG 6: An event generator for Hadron Emission Reactions with Interfering Gluons (including supersymmetric processes), JHEP 01 (2001) 010, [hep-ph/0011363].

[3] G. Corcella et al., HERWIG 6.5 release note, [hep-ph/0210213].

[4] S. Frixione and B. R. Webber, Matching NLO QCD computations and parton shower simulations, JHEP 06 (2002) 029, [hep-ph/0204244].

[5] S. Frixione, P. Nason, and B. R. Webber, Matching NLO QCD and parton showers in heavy flavour production, JHEP 08 (2003) 007, [hep-ph/0305252].

[6] P. Nason and G. Ridolfi, A positive-weight next-to-leading-order Monte Carlo for Z pair hadroproduction, JHEP 08 (2006) 077, [hep-ph/0606275].

[7] P. Nason, S. Dawson, and R. K. Ellis, The total cross-section for the production of heavy quarks in hadronic collisions, Nucl. Phys. B303 (1988) 607.

[8] P. Nason, S. Dawson, and R. K. Ellis, The one particle inclusive differential cross-section for heavy quark production in hadronic collisions, Nucl. Phys. B327 (1989) 49-92.

[9] M. L. Mangano, P. Nason, and G. Ridolfi, Heavy quark correlations in hadron collisions at next-to-leading order, Nucl. Phys. B373 (1992) 295-345.

[10] T. Sjostrand, S. Mrenna, and P. Skands, PYTHIA 6.4 physics and manual, JHEP 05 (2006) 026, [hep-ph/0603175].

[11] S. Frixione, P. Nason, and G. Ridolfi, The POWHEG-hvq manual version 1.0, arXiv:0707.3081 [hep-ph].

[12] R. Bonciani, S. Catani, M. L. Mangano, and P. Nason, Sudakov resummation of multiparton qcd cross sections, Phys. Lett. B575 (2003) 268-278, [hep-ph/0307035].

[13] S. Frixione, P. Nason, and C. Oleari, Matching NLO QCD computations with parton shower simulations: the POWHEG method, arXiv:0709.2092 [hep-ph].

[14] A. D. Martin, R. G. Roberts, W. J. Stirling, and R. S. Thorne, Uncertainties of predictions from parton distributions. i: Experimental errors. ((t)), Eur. Phys. J. C28 (2003) 455-473, [hep-ph/0211080].

[15] O. Latunde-Dada, S. Gieseke, and B. Webber, A positive-weight next-to-leading-order Monte Carlo for $e^{+} e^{-}$annihilation to hadrons, JHEP 02 (2007) 051, [hep-ph/0612281].

[16] S. Gieseke, A. Ribon, M. H. Seymour, P. Stephens and B. Webber, Herwig++ 1.0: An event generator for e+ e- annihilation, JHEP 0402 (2004) 005 [arXiv:hep-ph/0311208]. 